\title{
O enfermeiro frente ao paciente portador de insuficiência renal crônica
}

A Insuficiência Renal Crônica pode ser vista como um problema de saúde pública a nível mundial, isso se dá devido as suas elevadas taxas de incidência e prevalências, além do risco de morte. No Brasil, as taxas são altas e vem aumentado progressivamente, com tratamentos que apresentam elevados custos. Independente da causa da insuficiência, as complicações advindas dela comprometem ainda mais a qualidade de vida do paciente, gerando limitações, dependência, sentimentos frustrantes e sinais e sintomas indesejáveis. Entretanto, há que se fazer menção aos tipos de tratamentos existentes como diálise, hemodiálise e transplante renal, que por sua vez devolvem ao doente a esperança da melhoria da sua condição de saúde distanciando a ocorrência de desfechos indesejados. Prevenir é a melhor conduta, através de hábitos de vida saudáveis. A enfermagem possui papel fundamental, estimulando ações preventivas, prestando assistência ambulatorial, hospitalar e domiciliar ao indivíduo com a doença já instalada e a sua família por meio de procedimentos e educação em saúde, além de participar do desenvolvimento de protocolos em saúde voltados para essa classe de enfermidade.

Palavras-chave: Enfermeiro; Insuficiência Renal Crônica; Tratamento.

\section{The nurse facing the patient with chronic renal failure}

Chronic Kidney Failure can be seen as a public health problem worldwide, due to its high incidence rates and prevalence, as well as the risk of death. In Brazil, the rates are high and has been progressively increased, with treatments that present high costs. Regardless of the cause of the insufficiency, the complications arising from it further compromise the patient's quality of life, generating limitations, dependence, frustrating feelings and undesirable signs and symptoms. However, mention must be made of the types of treatments available, such as dialysis, hemodialysis and renal transplantation, which in turn give the patient hope of improving his health condition by distancing the occurrence of unwanted outcomes. Preventing is the best conduct through healthy living habits. Nursing has a fundamental role, stimulating preventive actions, providing outpatient, hospital and home care to the individual with the disease already installed and his family through procedures and health education, in addition to participating in the development of health protocols for this class of illness.

Keywords: Nurse; Chronic Renal Insufficiency; Treatment.

\section{Topic: Enfermagem Geral}

Reviewed anonymously in the process of blind peer
Received: $11 / 12 / 2017$

Approved: $15 / 01 / 2018$
Adriana Keila Dias

Faculdade Guaraí, Brasil

http://lattes.cnpq.br/2128882976477548

adrianakeiladias@hotmail.com

Reobbe Aguiar Pereira

Faculdade Guaraí, Brasil

http://lattes.cnpq.br/7447115724350334

reobbeap@hotmail.com

\section{Referencing this:}

DIAS, A. K.; PEREIRA, R. A.. O enfermeiro frente ao paciente portador de insuficiência renal crônica. Scire Salutis, v.8, n.1, p.25-36, 2018. DOI: http://doi.org/10.6008/CBPC2236-9600.2018.001.0004 


\section{INTRODUÇÃO}

Os rins são órgãos extremamente eficientes e essenciais para a manutenção da homeostase do corpo. Um ser humano pode viver normalmente possuindo apenas um rim, segundo relatos de doações de rins realizadas entre pessoas da mesma família, desde que ao menos um terço do rim seja funcional, é possível sobreviver, entretanto, quando há insuficiência renal, sem tratamento médico com hemodiálise, o óbito é inevitável (RIZZO, 2012).

A Insuficiência Renal Crônica (IRC) ou Doença Renal Crônica (DRC) é definida como perda progressiva e irreversível das funções renais e acomete cada vez mais as pessoas, seja em decorrência do processo de envelhecimento ou frente a elevação da quantidades de indivíduos portadores de hipertensão e diabetes mellitus (SIVIERO et al., 2014). A perda lenta e gradual do correto funcionamento dos rins é vista como a IRC, o dano que ocorre nesse órgão tende a se agravar por meses ou anos, ou seja, a função renal é comprometida.

A DRC é visualizada nas últimas décadas com um problema de saúde pública em consequência do aumento de sua prevalência entre a população mundial e ao seu impacto na morbimortalidade das pessoas portadoras. Resultante da crescente epidemia dos fatores de risco cardiovasculares, essa patologia resultará em internações frequentes e em grande gasto econômico. No ano de 2011, constatou-se 91.314 pacientes em terapia dialítica no Brasil, estima-se que 50 a $70 \%$ dos brasileiros que possuem a DRC terminal vão a óbito sem utilizar alguma modalidade de tratamento. O doente renal crônico tem mais chance de morrer de doença cardiovascular do que evoluir para a falência renal (PINHO et al., 2015).

O enfermeiro também desempenha uma função importante como educador em saúde, além do seu compromisso ético e profissional, sendo dessa forma um dos grandes responsáveis por estimular o autocuidado à saúde, visto que estar em constante contato com o paciente, assim, atuando na prevenção e retardo da progressão da insuficiência renal crônica (TORTORA et al., 2014).

Diante das dificuldades de um portador de IRA, pergunta-se, qual o papel da enfermagem frente a um paciente portador de IRC?. A pesquisa revela-se de grande importância, uma vez que a área da nefrologia que está inserida na saúde pública tem crescido muito nas últimas décadas, em decorrência das patologias crônicas e do aumento da expectativa de vida, o que acaba por demandar da enfermagem conhecimento apropriado para ofertar de modo efetivo a assistência ao indivíduo portador da insuficiência renal crônica nas unidades de saúde, assim como no âmbito domiciliar. O tema foi escolhido diante da necessidade de entender de quais formas ocorre a assistência de enfermagem aos pacientes com IRC, pois ao se deparar com pacientes acometidos por essa doença é possível notar como a enfermagem é essencial ao longo desse processo, visto que a IRC interfere na qualidade e expectativa de vida dos pacientes.

Diante do que foi referido, anteriormente, pode-se ressaltar a relevância da pesquisa relacionada a Insuficiência Renal Crônica. O enfermeiro deve possuir entendimento sobre esta doença em maior escala possível e com isso assistir da melhor forma possível ao paciente acometido, tanto no âmbito da assistência durante o tratamento como no de prevenção. 
Por meio da elaboração de tal estudo objetiva-se: descrever o papel do enfermeiro na assistência de enfermagem ao paciente portador de Insuficiência Renal Crônica, dentre outros, tais como: conceituar a fisiopatologia da IRC; destacar os principais sinais e sintomas clínicos da doença; discutir as medidas de prevenção para IRC; e relatar os métodos terapêuticos para amenizar os efeitos e avanço da doença.

\section{METODOLOGIA}

O trabalho foi desenvolvido com base em uma metodologia de pesquisa bibliográfica de caráter qualitativo, descritivo e exploratório. Serão utilizados livros, revistas e artigos. A pesquisa foi realizada durante os meses de fevereiro à junho de 2018.

\section{DISCUSSÃO TEÓRICA}

\section{Histórico}

A insuficiência renal crônica tem apresentado progressivamente altos índices de incidência e prevalência, anualmente em toda a esfera global. Esta patologia vem ainda acompanhada de alta morbidade e letalidade, evidenciando-se por perda lenta, contínua e irreversível das funções renais (RIELLA, 2010). No Brasil, a estimativa é que cerca de 120.000 indivíduos estejam em tratamento dialítico e com transplante renal, gerando um gasto aproximado de 1,4 bilhão de reais. Sem levar em consideração a doença base, os principais desfechos em pessoas portadoras de DRC são as suas complicações, tais como, desnutrição, anemia, comprometimento do metabolismo mineral e acidose metabólica que surgem em decorrência da perda da capacidade renal, além da falência funcional renal e do óbito (BASTOS et al., 2010).

A prevenção da insuficiência renal e a investigação com o intuito de detectar a síndrome no início é de suma importância para o retardo e complicações dessa doença, dando ao indivíduo chances de uma recuperação renal, evitando que ele seja direcionado para a terapia de substituição renal. Algumas pessoas, em particular, necessitam ser monitoradas de modo criterioso em relação à filtração glomerular, contudo são classificados como grupo de risco para o desenvolvimento de insuficiência renal. Tal grupo e composto por indivíduos portadores de diabetes, hipertensão arterial, de doenças cardiovasculares, de outras doenças renais, e indivíduos com histórico familiar de insuficiência renal (MONTEIRO, 2010).

Os rins que possuem a formal oval, retiram o excesso de água, sais e resíduos do metabolismo proteico do sangue, na medida em que devolvem nutrientes e substancias químicas ao sangue (MOORE et al., 2012).

Os rins formam um par de órgãos avermelhados, similares a feijões, localizados logo acima da cintura, entre o peritônio e parede posterior do abdômen. Devido apresentar posição posterior ao peritônio da cavidade abdominal, esse posicionamento e conhecido como retroperitoneal, localizam-se entre os níveis da última vértebra torácica e a terceira vértebra lombar, em uma posição onde permanecem parcialmente protegidos pelo décimo primeiro e décimo segundo pares de costelas. O rim esquerdo é ligeiramente mais alto que o direito, em virtude do fígado ocupar um espaço considerável, no lado direito, acima do rim. Eles 
medem cerca de 10 a $12 \mathrm{~cm}$ de comprimento, de 5 a $7 \mathrm{~cm}$ de largura e $3 \mathrm{~cm}$ de espessura, pesando cerca de 135 a 150 g. Cada rim é envolvido por três camadas de tecido, a camada profunda, denominada cápsula fibrosa, a camada média, denominada cápsula adiposa e camada superficial, denominada de fáscia renal. A unidade funcional dos rins é chamada de néfron, eles realizam as seguintes tarefas: filtração glomerular, secreção tubular e reabsorção tubular (TORTORA et al., 2014).

A IRC ocorre quando há presença de alterações na taxa de filtração glomerular e/ou existência de lesão parenquimatosa presentes por um período de três meses. A redução progressiva da função renal interfere no adequado funcionamento de vários outros órgãos. A função renal é avaliada pela filtração glomerular (FG) e a sua redução é verificada na IRC, relacionada a perda das funções regulatórias, endócrinas e excretórias dos rins, em situações onde a FG apresenta níveis muito reduzidos, abaixo de 15 $\mathrm{mL} / \mathrm{min} / 1,73 \mathrm{~m}^{2}$, ocorre o que designamos falência funcional renal (FFR), ou seja, o estágio mais avançado de perda funcional progressiva verificado na insuficiência renal crônica (BASTOS et al., 2010).

A insuficiência renal crônica é constatada como uma lesão renal com perda progressiva e irreversível da função renal (glomerular, tubular e endócrina). Nas fases mais avançadas (denominada de fase terminal de insuficiência renal crônica), os rins não possuem mais aptidão para equilibram a normalidade do meio interno do paciente (JUNIOR, 2004).

Os rins são responsáveis por realizarem o trabalho principal do sistema urinário, sendo funcionalidades dos rins: regulação do volume sanguíneo; regulação do pH sanguíneo; regulação da composição iônica do sangue; regulação da pressão arterial sistêmica; regulação da concentração sanguínea de glicose; manutenção da osmolaridade do sangue; produção de hormônios; excreção de resíduos e substâncias estranhas (TORTORA et al., 2014).

Existem três estágios da doença renal crônica: 1. Reserva renal reduzida - É determinada pela perda de 40 a 75\% da função do néfron, normalmente esta fase é assintomática, devido os néfrons remanescentes manterem a função renal; 2. Insuficiência renal - Acontece nos casos em que são perdidos 75 a $90 \%$ dos néfrons, onde a creatinina e ureia sérica apresentam-se elevadas, assim o rim perde aptidão de concentração de urina e a anemia surge, sendo que o indivíduo pode apresentar poliúria e nictúria; 3. Doença renal em estágio terminal - O estágio final da IRC ocorre em situações onde restam menos de $10 \%$ da função dos néfrons, onde todas as funções reguladoras, secretoras e excretoras do rim normal estão afetadas, além que nesta fase é comprovada pelo aumento sérico de ureia e creatinina, assim como pela falta de equilíbrio hidroeletrolítico, por isso que neste caso, a diálise é recomendada (SMELTZER, 2011).

A insuficiência renal crônica (IRC) é definida por lesão renal quando há perda progressiva e irreversível da função dos rins, o que leva à perda da aptidão de conservar o equilíbrio metabólico e hidroeletrolítico (CUNHA, et al., 2009). Na fase avançada da IRC, os túbulos glomerulares, interstício e vasos encontram-se esclerosados, assim, see essa desordem não for devidamente tratada, com diálise ou transplante, poderá ocorrer óbito por uremia (KUMAR et al., 2013). 


\title{
Causas e Sintomas
}

São inúmeras as etiologias da IRC, entre as quais: hipertensão arterial sistêmica, diabetes mellitus, processos renais obstrutivos crônicos, pielonefrite, amiloidose, necrose cortical renal grave, glomerulonefrite crônica, rins policísticos, entre outras. Hipertensão e diabetes, descontroladas ou em formas graves, são as mais frequentes causas que levam os pacientes a IRC, sendo necessário diálise e/ou transplante renal (PRADO et al., 2007).

\begin{abstract}
A gravidade dos sinais e sintomas da IRC depende do grau de comprometimento renal e da idade do paciente. Essas manifestações aparecem em todos os sistemas do organismo pela presença da uremia. São observadas manifestações neurológicas centrais e periféricas; alterações gastrintestinais, endócrinas, metabólicas, infecciosas, dermatológicas e hematológicas. Essas alterações, em conjunto, podem levar o paciente à fadiga e à dispneia. A IRC somada ao tratamento hemodialítico é igual à possibilidade da presença de várias complicações, como deterioração musculoesquelética, fraqueza, descoloração da pele, emagrecimento, edema, fadiga e alterações pulmonares. (CUNHA, et al., 2009)
\end{abstract}

A desnutrição é uma condição presente comumente em doentes renais crônicos e está associado a perda de massa corporal, hipercatabolismo de proteínas e perda ou diminuição de energia, sendo ainda, resultante de restrições graves na alimentação, consumo insuficiente de nutrientes, distúrbios do trato gastrointestinal e hormonal, acidose metabólica, remédios que interferem na absorção de alimentos, além de condições relacionadas a terapêutica, como perda de nutrientes no momento do procedimento dialítico e dialise imprópria (SIVIERO et al., 2014).

As principais complicações desenvolvidas a partir da redução ou perda das funções renais são: diminuição ou perda do olfato e paladar; anorexia; uremia; desnutrição; hiperglicemia; hipertensão e hipertrigliceridemia, que podem desencadear complicações cardiovasculares; e ainda propensão a hemorragia devido a incapacidade renal; desorientação; edema; oligúria; adinamia; obnubilação; asterixe e coma (SANTOS, et al., 2013).

Pode-se afirmar que o estresse causado pela doença renal compromete a autoestima, a relação familiar e conjugal, além de afetar vários outros aspectos do cotidiano do indivíduo portador desta complicação crônica. No decorrer da redução da função renal o paciente evolui para a insuficiência renal crônica, geralmente apresentado falência de múltiplos órgãos, podendo ocasionar sequelas como, redução ou ausência da acuidade visual, impotência e insuficiência cardíaca (MASCARENHAS, 2011).

As mais frequentes etiologias da Insuficiência Renal Crônica (IRC) são: a diabetes mellitus, a hipertensão arterial sistêmica, doenças renais (glomerulopatia, nefropatia tubulointerticial, doença renal policística, displasia, hipoplasia renal) e uropatias (infecções urinárias repetitivas, obstruções urinárias e cálculos urinários) (SANTANA et al., 2013).

\section{Tratamento}

Após ser diagnosticado com IRC o paciente primeiramente receberá ao tratamento conservador, composto por administração de medicamentos e modificações nutricionais. De acordo com a progressão da doença o paciente será submetido a outros métodos terapêuticos, entre os quais: diálise peritoneal, 
hemodiálise e transplante de rim, mas para decidir a melhor opção de terapêutica é necessário examinar as condições clínicas, psicológicas e financeiras do indivíduo (LATA, et al., 2008).

Entre as principais formas de terapêuticas existentes estão a hemodiálise, diálise peritoneal e o transplante renal, o transplante é a opção mais utilizada de tratamento nos casos de insuficiência renal crônica, quando comparado as outras modalidades terapêuticas, isso se dá por oferecer menor custo e elevada qualidade de vida para o portador ao longo do tempo. A estimativa de pacientes em diálise no mês de julho de 2012 foi de 97.586 sendo que deste total 30.447 deles estavam inscritos em lista de espera para realização de transplante, o que equivale a um percentual próximo de 31,2\%. No ano de 2014, houve à realização de 4.255 transplantes renais advindos de doadores falecidos e 1.384 transplantes a partir de doadores vivos, o que equivale a um total de 5.639 transplantes ocorridos em território brasileiro (PAULETO, et al., 2016). Segundo Bastos et al. (2011), "o tratamento adequado na IRC é formado por três pontos principais: 1) diagnóstico precoce da IRC; 2) encaminhamento direto para tratamento nefrológico; 3) implementação de ações com a finalidade de manter a função renal". A terapêutica a ser instituída demanda o reconhecimento de aspectos distintos, entretanto associados, que enquadram a patologias de base, o estágio em que a patologia se encontra, a velocidade da redução da filtração glomerular, reconhecimento de complicações e comorbidades, especialmente as cardiovasculares (TORTORA et al., 2014).

O tratamento irá depender da evolução da patologia, podendo ser conservador através do uso de medicamento, dietético e restrição hídrica, quando esse tipo de tratamento não se mostrar eficaz, deve-se dar início a hemodiálise, diálise ou dialise peritoneal que substitui em parte a função a ser desempenhada pelos rins, ou se submeter a um transplante renal. Dentre estes, o mais frequente é a hemodiálise que constitui um processo de filtragem e depuração sanguínea de substancias indesejáveis como a ureia e a creatinina que precisam ser eliminadas da corrente sanguínea, pois em pacientes portadores de IRC este processo natural para de ocorrer (RODRIGUES et al., 2012).

O acompanhamento dos indivíduos doentes deverá ser feito por uma equipe multiprofissional, composta no mínimo por médio, enfermeiro, assistente social, psicólogo e nutricionista. O tratamento dos fatores de risco modificáveis para a progressão da IRC e doença cardiovascular deve ser seguido conforme as recomendações do Ministério da Saúde: controle da hipertensão arterial, da glicemia, obesidade, doenças cardiovasculares, da dislipidemia, tabagismo, etilismo e mudanças favoráveis dos hábitos de vida. E avaliação nefrologia conforme prescrição médica ou protocolo (BRASIL, 2014).

Na IRC o indivíduo é direcionado para o tratamento renal substitutivo tal como, diálise peritoneal, hemodiálise ou ainda transplante renal. Tais modalidades de tratamentos são indicadas quando o funcionamento renal se mostra inferior a 10-12\%. É uma doença que afeta a função renal do paciente, acarretando mudanças, como alterações físicas, psicológicas e sociais, mas a prevalência anual de pessoas em diálise tem aumentado progressivamente (SESSO, et al., 2011).

A diálise pode ser feita através hemodiálise ou diálise peritoneal. O modo comumente mais utilizado é a hemodiálise, no qual o sangue passa pela membrana semipermeável do rim artificial e os produtos de excreção são eliminados por difusão e os líquidos por ultrafiltração. A hemodiálise necessita de acesso 
contínuo a corrente sanguínea através de uma fístula feita por meio cirúrgico com o objetivo de conectar uma artéria a uma veia. Geralmente as fístulas são criadas próximas ao punho, o que eleva bastante o calibre das veias do antebraço, assim quando os vasos sanguíneos do paciente forem fracos, um vaso artificial denominado de enxerto pode ser cirurgicamente implantado, mas as agulhas de grosso calibre são inseridas na fístula ou enxerto antes de iniciar a diálise e removidas após o término da diálise. O acesso temporário por meio de cateteres subclávios é frequente até que o acesso permanente do paciente seja feito ou possa maturar, contudo, os riscos de infecções tornam estes cateteres indesejáveis. Os produtos de excreção e os eletrólitos se movem por difusão, ultrafiltração e osmose do sangue para o dialisado e são removidos, por essa razão, o processo hemodialítico dura em torno de 3 a 5 horas e ocorre normalmente três vezes por semana (MAHAN et al., 2005).

A diálise é um processo mecânico e artificial aplicado para remover do sangue as impurezas acumuladas pelo organismo. Os tóxicos que necessitam ser eliminados do organismo são removidos por meio de uma membrana filtrante de rim artificial ou do peritônio. Há duas formas de diálise: a Peritoneal que usa o peritônio como membrana filtrante e a Hemodiálise que utiliza uma membrana artificial para filtrar (FIDELIS, et al., 2016).

Dentre as formas de tratamento, a hemodiálise desponta quantitativamente, sendo que $70 \%$ dos portadores de IRC a nível mundial são submetidos a este tipo de terapia renal substitutiva. A máquina de hemodiálise desempenha a função de um rim artificial, removendo do sangue as toxinas e os resíduos nitrogenados, para logo após ser devolvido ao indivíduo, assim esse tratamento é feito, normalmente, três vezes na semana, com aproximadamente quatro horas de duração por sessão, com isso, a hemodiálise promove a sobrevivência do paciente renal crônico (FRAZÃO, et al., 2016).

\section{Assistência de Enfermagem}

Para a obtenção de resultados satisfatórios, pode-se enunciar as seguintes estratégias: confirmação do diagnóstico precocemente, imediato encaminhamento e estabelecimento de medidas que visem a redução ou cessação da evolução da doença. Uma vez em andamento, há indícios de que a assistência interdisciplinar, integral, organizada e abrangente, é a mais adequada tática para diminuir os índices de mortalidade. É fundamental haver empenho para colocar em prática as medidas sistemáticas e direcionadas de prevenção e controle das complicações advindas do tratamento e colaborar expressivamente para melhoria da qualidade de vida do portador (SIVIERO et al., 2014).

Prevenção é tratar e controlar os fatores de risco modificáveis: diabetes, hipertensão, dislipidemia, obesidade, doença cardiovascular e tabagismo, cujo controle e tratamento necessitam estar conforme as normatizações e orientações do Ministério da Saúde, mas, em se tratando dos medicamentos, aconselha-se que o uso crônico de qualquer tipo de medicação deve ser feito somente com orientação médica e precisase ter cuidado específico com agentes, que apresentem efeito nefrotóxico (CUNHA, et al., 2009).

É papel da enfermagem é orientar o paciente e a família sobre todo o procedimento de hemodiálise: duração, técnicas aplicadas, intercorrências indesejáveis que possam ocorrer, condutas e recursos dos 
equipamentos. Eles devem ainda serem aconselhados quanto ao cuidado necessário com o acesso vascular de modo a conservá-lo pérvio e livre de infecção (LASELVA et al., 2010).

As intercorrências mais comuns durante a hemodiálise são: hipotensão e hipertensão arterial, cefaleia, náuseas, arritmias, vômitos, algia na lombar e torácica e prurido. As menos constantes incluem: hipersensibilidade, hemorragias, hemólise, convulsões, embolia gasosa, reações pirogênicas e hipoxemia. Essas complicações podem ocorrer casualmente. Porém, dependendo da situação do paciente elas podem levar ao óbito, o que torna evidente a precisão de uma assistência de enfermagem alerta e eficaz no decorrer da terapia dialítica (DALLÉ et al., 2012).

O paciente renal crônico deve receber informações adequadas ao novo modo de vida que terá de assumir, com as rotinas das sessões de hemodiálise, a dieta alimentar, o cuidado com a higiene, dentre outros. Dessa forma, torna-se necessário julgar as respostas dessa clientela através do cuidado do profissional de enfermagem. Isso nos reporta à sistematização da assistência de enfermagem que contribui para a organização do trabalho do enfermeiro e para um melhor relacionamento com o paciente, proporcionando assim melhor norteador do cuidado prestado pelo enfermeiro a essa clientela. A hemodiálise é um tratamento paliativo, pois não recupera integralmente a saúde do paciente, ocasionando desgaste físico, estresse mental e emocional. 0 enfermeiro, por meio do diagnóstico de enfermagem, pode sistematizar seu trabalho e oferecer um cuidado de qualidade e individualizado a essa clientela. (LATA et al., 2008)

Antigamente, o tratamento hemodialítico era realizado pela equipe médica, entretanto com o passar dos anos, a enfermagem começou a participar ativamente do tratamento de terapia substitutiva renal, responsabilizando-se por toda a parte técnica e de relação do cliente com o meio externo. Por se tratar de um processo que origina várias complicações tais como: hipotensão, hipertensão, câimbras musculares, calafrios, febre, náusea, vômitos, cefaleia e prurido; faz-se essencial a atuação do Enfermeiro e da equipe de Enfermagem nestes casos, incluindo prestação de cuidados diretos e indiretos. A assistência de enfermagem representa o cuidado prestado com a qualidade que se almeja de um bom profissional, não apenas no caráter ético e humano, assim como da questão técnico-científico. Aplicando o Diagnóstico de Enfermagem como ferramenta de trabalho, expressando assim as necessidades de cuidados identificados, realizando o julgamento clínico sobre as respostas do paciente, da família e da coletividade aos processos de saúde (MELO, et al., 2015).

O tratamento hemodialítico é um processo que pode ocasionar várias complicações, tais como: câimbras, cefaleia, náuseas, dor torácica e lombar, hipotensão, hipertensão, vômitos, calafrios, prurido e febre, frente a tais intercorrências é fundamental o papel do enfermeiro e da equipe de enfermagem no controle de tais situações, dispensando ao paciente cuidados diretos e indiretos (MELO, et al., 2015).

O papel do enfermeiro perante a prevenção e progressão da IRC acontecerá a partir do conhecimento das reais necessidades dos portadores, assim é de suma importância que o enfermeiro esteja apto para detectar os grupos de risco, assim como identificar os indivíduos com a doença já instalada e partir daí traçar um plano de cuidados conforme cada caso clínico, pois ele deverá promover ações que visem a promoção da saúde através da adoção de hábitos de vida mais saudáveis e exclusão de costumes prejudiciais para o paciente que ocorrerá através da educação, saúde e orientação. Deverá também conscientizar o 
cliente das possíveis complicações que podem surgir no decorrer da doença. Poderá ainda traçar um plano assistencial que vise reduzir ou solucionar problemas que possam surgir durante a diálise (MELO et al., 2014).

A assistência de enfermagem engloba a sistematização desde a entrada do paciente até a saída deste da sessão de hemodiálise, devendo-se recepcionar o paciente ao chegar à unidade de diálise, observando sua condição geral de saúde e realizando uma avaliação pré-hemodiálise, que abrange encaminhamento do paciente à balança para registrar o peso, encaminhar o paciente à máquina, verificar sinais vitais; auxiliares e/ou técnicos devem informar qualquer alteração para o enfermeiro responsável, dialogar com o paciente a respeito de qualquer sintoma que ele tenha percebido desde a última diálise, etc. e se não houver restrição iniciar a sessão de diálise. Na avaliação pós-hemodiálise deve-se atentar para sinais de sangramento no local da punção venosa, verificar sinais vitais, constatar o peso e não permitir que o paciente sintomático saia da unidade sem passar por atendimento médico, etc. (SANTANA et al., 2013).

Devido as perdas das funções renais na IRC o indivíduo tem que se submeter a hemodiálise que consiste no desvio do sangue do organismo, por meio de um dialisador, onde ocorrerá a disfunção por ultrafiltração, e retorno posterior ao organismo. Tal procedimento é muito complexo para o paciente, requerendo dele mudanças intensas tanto da condição física, psicológica, etc., e isso torna a assistência de enfermagem indispensável durante o tratamento, pois compete a este profissional avaliar o paciente, o acesso venoso, dar assistência enquanto o cliente dialisa até estimulá-lo a aceitar as modificações no seu estilo de vida (SANTANA et al., 2013).

É imprescindível que o profissional de enfermagem esteja atento às queixas e reações ocorridas no paciente, deste modo ele poderá tomar medidas que amenizem as necessidades verificadas. A prática da educação em saúde em conjunto com uma assistência humanizada podem favorecer resultados positivos na qualidade de vida de seus pacientes e familiares. Há também que se fazer menção a necessidade do trabalho contínuo de uma equipe multiprofissional para a existência de uma assistência qualitativa e o mais distante de riscos possíveis (FIDELIS et al., 2016).

É importante salientar que o transplante requer uma rigorosa adesão ao tratamento. A ausência de cuidados voltados para a saúde pós-transplante, como o esquecimento da medição imunossupressora, pode acarretar problemas e comprometer o funcionamento eficaz do procedimento. Em relação a utilização de medicamento imunossupressor, uma investigação com pacientes transplantados verificou que $68 \%$ dos entrevistados referiram saber a relevância da administração correta dos medicamentos para a manutenção satisfatória do enxerto, já 32\% não reconheciam tal importância. A falta de adesão ao tratamento imunossupressor gera inúmeros riscos à saúde do paciente, sendo ainda um forte fator de causa de rejeição de enxerto. Diante disso, o profissional de enfermagem desempenha um papel essencial nas orientações de alta do paciente transplantado, pois este processo de educação em saúde representa um dos pilares de sustentação para aderir aos cuidados indispensáveis durante esta nova fase da vida que se inicia (SILVA et al., 2011).

É essencial a utilização de uma linguagem de fácil compreensão, simples e clara e sem aplicação de termos técnicos, por parte dos profissionais em saúde, quando esta comunicação for voltada para o paciente, 
com o intuito de explicar ao paciente todas as informações que são relacionadas ao seu tratamento. 0 enfermeiro deve tirar proveito do tempo que passa com o paciente nas sessões de hemodiálise para aumentar vínculos e, com isso, sanar questionamentos duvidosos do paciente (KNIHS, et al., 2013).

O enfermeiro desempenha papel fundamental nas ações de prevenção e controle, uma entre muitas de suas funções é prestar assistência a pacientes na avaliação diagnóstica, tratamento, reabilitação e atendimento aos familiares. Deve ainda realizar ações educativas, ações conjunta com outros profissionais, apoiar medidas legislativas e identificar fatores de risco ocupacional, no contexto da assistência ao portador da IRC, em tratamento hemodialítico, por isso, a pesquisa enfermagem sobre esse tema é fundamental para proporcionar a base de conhecimento que fundamenta a prática clínica, sendo possível ainda identificar o impacto da IRC e hemodiálise no tratamento e na vida do portador e sua família. O enfermeiro é o eixo central no contato com os pacientes, isso evidencia o quão valoroso é sua atuação na pesquisa, qualificação e conhecimento, multiplicando as informações para a equipe de enfermagem e qualificando o processo de assistência. A pesquisa pode ser determinada como uma ferramenta de crescimento, avanço e valorização da profissão na sociedade e não somente um método utilizado para obter conhecimento para a prática diária, mas visando a enfermagem como ciência (RODRIGUES et al., 2012).

\section{CONCLUSÕES}

A IRC apresenta elevados índices de incidência e prevalência o que acaba por acarretar um elevado custo para o sistema de saúde, assim como para os indivíduos que optam por tratamento particular. Além de interferir negativamente na qualidade de vida, pois são várias as complicações que podem surgem devido a sua existência, sendo ainda considerada um problema de saúde pública a nível mundial.

Os rins possuem importante função no nosso organismo, por isso é de suma importância detectar a doença em seu estágio inicial para que o médico institua uma terapêutica e esta seja iniciada imediatamente favorecendo assim um melhor prognóstico para o paciente. A Insuficiência Renal Crônica (IRC) também denominada como Doença Renal Crônica (DRC) é um agravo a saúde que geralmente ocorre secundariamente a outra doença, ou seja, é tida como uma complicação, sendo que muitas vezes também desencadeará o surgimento de outras patologias, tais que podem ocorrer por várias causas, entre as quais, obstrução, infecção, secundariamente a outras doenças, defeitos genéticos, nefrolitíase e glomerulonefrite, tudo isso podendo gerar insuficiência renal crônica.

Boa parte dos pacientes apresentam pensamentos negativos quando são transplantados e mesmo assim são retornados novamente para hemodiálise. Diante disso, ressalta-se a necessidade dos indivíduos em tratamento dialítico possuírem entendimento relacionado a duração do transplante e que ele depende do tipo de doador e dos aspectos clínicos de cada pessoa. Será necessário acompanhamento contínuo, utilização de medicamentos imunossupressores além de cuidados voltados para a manutenção da saúde física e psíquica, sendo todas estas informações podem ser repassadas pela equipe de saúde responsável bem como a assistência. 
Os pacientes renais crônicos veem no transplante renal a solução para os seus problemas, ou seja, suas expectativas quanto ao futuro são positivas, pois esperam a partir dele a chegada de uma vida nova, com mais independência e segurança favorecendo a melhoria da qualidade de vida. Contudo, há ainda o medo devido a desconfiança quanto ao sucesso e durabilidade do transplante, com isso, faz-se necessário a qualificação profissional do enfermeiro, devendo ele ser conhecedor das expectativas pessoais do paciente acerca do transplante renal ao qual será submetido, a fim de que isso favoreça a qualidade e eficácia na prestação de assistência as necessidades individuais do cliente.

Com o intuito de prevenir a IRC, recomenda-se, a prática regular de algum tipo de atividade física aliada a manutenção satisfatória do peso corporal, controlar os níveis de açúcar no sangue com a finalidade de impedir a diabetes, monitoramento da pressão arterial sistêmica, aderir a uma alimentação saudável e balanceada, manter-se hidratado, evitar o tabagismo e etilismo, além da realização de exames preventivos que podem ser solicitados durante a consulta médica.

Prevenção é a melhor opção, porém na maioria das vezes essa ação é ignorada pelas pessoas que não dão importância a esta simples prática, prevenir é primordial, anda mais frente a casos de indivíduos que já são potencialmente propensos a desenvolver essa enfermidade por já possuírem doença ou fatores predisponente. As medidas de prevenção são globais, ou seja, recomendadas a todos, entre elas, redução ou abdicação do consumo de álcool e tabaco, não exagerar na automedicação, praticar atividade física, alimentação saudável, manutenção do peso adequado, manter a pressão arterial sistêmica em níveis satisfatórios, além de outras medidas.

\section{REFERÊNCIAS}

BASTOS, M. G.; BREGMAN, R.; KIRSZTAJN, G. M.. Doença renal crônica: frequente e grave, mas também prevenível e tratável. Rev. Assoc. Med. Bras., 2010.

BASTOS, M. G.; KIRSZTAJN, G. M.. Doença renal crônica: importância do diagnóstico precoce, encaminhamento imediato e abordagem interdisciplinar estruturada para melhora do desfecho em pacientes ainda não submetidos à diálise. Jornal Brasileiro de Nefrologia, 2011.

BRASIL. Ministério da Saúde. Diretrizes Clínicas para o Cuidado ao paciente com Doença Renal Crônica - DRC no Sistema Único de Saúde. Brasília: Ministério da Saúde, 2014.

CUNHA, M. S.; ANDRADE, V.; GUEDES, C. A.; VELOSO, M. C. H. Z.; AGUIAR, A. P.; CARDOSO, A. L.. Avaliação da capacidade funcional e da qualidade de vida em pacientes renais crônicos submetidos a tratamento hemodialítico. Revista Fisioterapia e Pesquisa, 2009.

DALLÉ, J.; LUCENA, A. F.. Diagnósticos de enfermagem identificados em pacientes hospitalizados durante sessões de hemodiálise. Acta paul. enferm., 2012.

FIDELIS, C. I.; BALBINO, C. M.; SOUZA, M. M. T.; RODRIGUES, L. M. S.; SILVINO, Z. R.; PASSOS, J. P.. Dificuldades enfrentadas pelo paciente renal para a realização do tratamento. Revista enfermagem atual in derme, 2016.
FRAZÃO, C. M. F. Q.; TINÔCO, J. D. S.; FERNANDES, M. I. C. D.; MACEDO, B. M.. Modificações corporais vivenciadas por pacientes com doença renal crônica em hemodiálise. Enfermería Global, 2016.

JUNIOR, J. E. R.. Doença Renal Crônica: Definição, Epidemiologia e Classificação. Jornal Brasileiro de Nefrologia, 2004.

KNIHS, N. S.; SARTORI, D. L.; ZINK, V.; ROZA, B. A.; SCHIRMER, J.. A vivência de pacientes que necessitam de transplante renal na espera por um órgão compatível. Texto \& Contexto - Enfermagem, 2013.

KUMAR, V.; ABBAS, A. K.; ASTER, J. C.. Robbins Patologia Básica. 9 ed. Rio de Janeiro: Elsevier, 2013.

LASELVA, C. R.; JÚNIOR, D. F. M.. Terapia intensiva: enfermagem. São Paulo: Editora Atheneu, 2010.

LATA, A. G. B.; ALBUQUERQUE, J. G.; CARVALHO, L. A. S. B. P.; LIRA, A. L. B. C.. Diagnósticos de enfermagem em adultos em tratamento de Hemodiálise. Acta Paulista de Enfermagem, 2008.

MAHAN, L. K.; ESCOTT-STUMP, S.. Alimentos, nutrição e dietoterapia. 11 ed. São Paulo: Roca, 2005. 
MASCARENHAS, N. B.; PEREIRA, A.; SILVA, R. S.; SILVA, M. G.. Sistematização da Assistência de Enfermagem ao portador de Diabetes Mellitus e Insuficiência Renal Crônica. Rev. Bras. Enferm., 2011.

MELO, W. F.; BEZERRA, A. L. D.; SOUSA, M. N. A.. Perfil epidemiológico de pacientes com insuficiência renal crônica: um estudo quantitativo. Revista Eletrônica da Fainor, 2014.

MELO, W. F.; PEREIRA, A. W. R.; ALVES, V. Q.; SALDANHA, H. G. A. C.; SOUSA, J. S.. Assistência de enfermagem na urgência e emergência ao paciente vítima de Insuficiência Renal Aguda: uma revisão bibliográfica. Revista Brasileira de Educação e Saúde, 2015.

MONTEIRO, E. F.. Metodologia de pesquisa na engenharia de produção e sistemas. Revista das Faculdades Santa Cruz, 2010.

MOORE, K. L.; DALLEY, A. F.; AGUR, A. M. R.. Anatomia orientada para a clínica. 6 ed. Rio de Janeiro: Guanabara Koogan, 2012.

PAULETO, M. R.; BEUTER, M.; TIMM, A. M. B.; SANTOS, N. O.; ROSO, C. C.; JACOBI, C. S.. Transplante renal: percepção de pacientes em hemodiálise fora da lista de espera. Rev. Enferm. UFSM, 2016.

PINHO, N. A.; SILVA, G. V.; PIERIN, A. M. G.. Prevalência e fatores associados à doença renal crônica em pacientes internados em um hospital universitário na cidade de São Paulo. Jornal Brasileiro de Nefrologia, São Paulo. 2015.

PRADO, F. C.; RAMOS, J.; VALLE, J.. Atualização Terapêutica. São Paulo: Artes Médicas, 2007.
RIELLA, M. C.. Princípios de nefrologia e distúrbios hidroeletrolíticos. 5 ed. Rio de Janeiro: Guanabara Koogan, 2010.

RIZZO, D. C.. Fundamentos de anatomia e fisiologia. São Paulo: Cengage Learning, 2012.

RODRIGUES, I. G.; NAKAHATA, K. S.. Estudos de enfermagem sobre a Doença Renal Crônica. Rev. Enferm. UNISA, 2012.

SANTANA, S. S.; FONTENELLE, T.; MAGALHÃES, L. M.. Assistência de enfermagem prestada aos pacientes em tratamento hemodialítico nas unidades de nefrologia. Revista Científica do ITPAC, 2013.

SESSO, R. C.; LOPES, A. A.; THOMÉ, F. S.; SANTOS, J. R. L. D. R.. Relatório do censo brasileiro de diálise. Jornal Brasileiro de Nefrologia, 2011.

SILVA, J. M.; FIALHO, A. V. M.; BORGES, M. C. L. A.; SILVA, L. M. S.. Perfil epidemiológico dos pacientes transplantados renais em hospital universitário e o conhecimento sobre uso de drogas imunossupressoras. J. Bras. Transpl., 2011.

SIVIERO, P. C. L.; MACHADO, C. J.; CHERCHIGLIA, M. L.. Insuficiência renal crônica no Brasil segundo enfoque de causas múltiplas de morte. Cad. saúde colet., 2014.

SMELTZER, S. C.. Tratado de Enfermagem Médico-Cirúrgica. 12 ed. Rio de Janeiro: Guanabara Koogan, 2011.

TORTORA, G. J.; DERRICKSON, B.. Princípios de anatomia e fisiologia. 12 ed. Rio de Janeiro: Guanabara Koogan, 2014.

A CBPC - Companhia Brasileira de Produção Científica (CNPJ: 11.221.422/0001-03) detém os direitos materiais desta publicação. Os direitos referem-se à publicação do trabalho em qualquer parte do mundo, incluindo os direitos às renovações, expansões e disseminações da contribuição, bem como outros direitos subsidiários. Todos os trabalhos publicados eletronicamente poderão posteriormente ser publicados em coletâneas impressas sob coordenação da Sustenere Publishing, da Companhia Brasileira de Produção Científica e seus parceiros autorizados. Os (as) autores (as) preservam os direitos autorais, mas não têm permissão para a publicação da contribuição em outro meio, impresso ou digital, em português ou em tradução. 